\title{
Reality, Information, and Information Observer ${ }^{\dagger}$
}

\author{
Vladimir S. Lerner \\ Independent researcher, 13603 marina pointe drive, Marina Del Rey, CA 90292, USA; lernervs@gmail.com; \\ Tel.: +1-310-8226279 \\ + Conference Theoretical Information Studies (TIS), Berkeley, CA, USA, 2-6 June 2019.
}

Published: 20 May 2020

\begin{abstract}
Up to now both information and its connection to reality do not have scientifically conclusive definitions, nor implicit origin. They emerge in observing multiple impulses' interactive yes-no actions modeling information bits. Each memorized bit of information binds a reversible microprocess with an irreversible information macroprocess along a multi-dimensional observing process. Interactive information dynamics assemble geometrical and information structures of observer cognition and intelligence in double spiral rotating code. Information Path Functional integrates multiple interactive dynamics in finite bits which observe and measure reality. The time and space of reality exists only as discrete units of information. The observed information process, creating its Observer, connects reality, information, and the Observer.
\end{abstract}

Keywords: reality; interaction; impulse; interactive observation; logic; certainty; information process; micro-macroprocess; Information Path Functional; triple structural unit; triplet code

\section{Introduction}

Observers are everywhere, from communicating people, animals, and different species up to any interacting subjects, accepting, transforming, and exchanging information.

What do the observers actually observe? Do they observe reality? What is the information they observe? And how is the observed information connected with the reality of observation? What is the scientific path to uncovering the fact of reality through its observed information?

All these questions are still unanswered in known publications.

In [1] the eminent scientists discussed various aspects of information and reality, from quantum information to biological and digital information, in order to understand how nature works.

Most discussions focus on unproven physical phenomena, such as multiple universes, "everywhere is conscience", complexity emerging through cosmological and biological evolution, and others whose origin is still unknown. Even evolution is an interacting process producing all these phenomena.

However, a concept "that all reality is information and all information is reality, and the universe is composed of bits of information" is close to ours.

But how the bits of information emerge from reality is not discussed.

According to concept [2]: "It from bit symbolizes the idea that every item of the physical world has at bottom - at a very deep bottom, in most instances - an immaterial source and explanation; that which we call reality arises in the last analysis from the posing of yes-no questions". This concept is most closely related to ours. We add that "information bits model multiple yes-no actions."

Inter-actions unify all yes-no actions in physics and human beings' behavior. That is why reality is a dynamic phenomenon of interactions, which create information.

Anther concept, [3], is that "information makes no sense in the absence of something to be informed - that is, a conscious observer capable of choice, or free will." 
We agree that information should be observed, adding concept observation and its observer to our concept.

For example, when searching for information on the World Wide Web, a potential observer sends probing impulses, interacting with World Wide Web observing events and activating his or her brain-neuron impulses until actual informational fact appears for the observer.

This observer becomes the observer of this information or the Information Observer.

Interaction is fundamental reality building the structure of the Universe from deep levels of physics: four fundamental interactions (gravitation, electromagnetic, weak, and strong) toward different chemical, thermodynamic, biological, human observational, up to brain neurons' interactive actions, cognitive, intellectual, and communicational-in all forms of life. They evolve in various substances of our world as interconnection is binding them.

Some reveal reality as actuality (which acts or interacts). For many of these objects, reality is a permanent dynamic state running interaction and producing information. The memorized and encoded information frizzes the interactive reality level. Where there is interaction, there is a reality and information transferring through the interaction. Any interaction is actual or virtual.

This leads to the concept of observing information in interacting reality.

However, up to now both information and its connection to reality do not have scientifically conclusive definitions, nor implicit origin, and interactions have not been considered as phenomena of reality.

The connection of Reality-Information-Information-Observer is still unexplained.

What is the scientific path to the fact of reality through its observed information?

Is reality continuous or discrete like $0 \mathrm{~s}$ and $1 \mathrm{~s}$ of information?

The scientific path emerges in observing multiple impulses' interactive yes-no actions modeling information bits. Multiple interactions build the Universe independently of their origin, and reality is only the emerging interactions. Alternatively, information is a phenomenon of interactions bringing a certain reality with the information of observing bits encoding an image.

Multiple interactions are random composing random process which covers both the interacting bits and the process certainty. Uncovering the bits and the information process, carrying certainty through the observation of the random process, runs an Observer. Otherwise, the observation of the information carried reality creates a certain information process from which the Information Observer emerges.

The observed information creating its Observer connects reality, information, and Observer.

\section{Foundation of the Approach}

\subsection{Axiom}

Multiple interactions build the Universe independently of their origin, and reality is only the emerging interactions.

Reality is an objective abstract entity only as a universal interaction.

Inter-action is interconnection that includes act of probing, or observing reality.

The observed interaction creates a specific certain reality.

\subsection{Corollaries}

1. Natural interactions unify a sequence of interactive impulses' Yes-No (or No-Yes) actions.

Each real (certain) interaction is opposite a yes-no action modeling an elementary bit of information or a discrete impulse of $1|0,0| 1$ qubit.

Information is a phenomenon of interactions which also brings reality.

2. Bringing reality, as interactions carrying information, requires the observation conveying the impulse information. The interaction of multiple impulses is source of randomness, which model series of random impulses. Multiple interactive impulses compose random processes in surrounding random field [4:26]. The field unifies the universal interactions. The field 
connects sets of possible and actual events with their probabilities. This connection forms a triad. Each triad emanates the events random process of natural interactions.

3. Each observation is act changing an observing process that generates an interactive observation. A manifold of observing actions identifies particular observer which observes the specific triad' random process. The process' random states (events) conserve energy of actual (real) events, whose interacting impulses covers information bits.

4. Uncovering a real bit and/or multiple bits or qubits from a random process provides the observation process of probabilistic interactive impulses. Disclosing the bit's discrete impulses requires interactive cuts of the random process during a virtual probabilistic impulse observation.

5. When reaching the observing certain reality the interactive process probability runs to approaching one, then the real cut delivers the impulse high-quality (quantum) energy.

This exposes the bit emerging from the observing specific impulse interactions as a unit of information with the information process carrying an explicit image and a certain reality, creating an Information Observer with a Net of assembling multi-level interactive information units.

Information emerges as a phenomenon of interactions and an observed measure of the interactions.

\section{Essence of Mathematical Modeling Methodology Implementing the Axiom and Corollaries}

The modeling uses example with an astronomer that traces unobserving planet image by its probabilistic trajectory until it become most probable by registering a memorizing copy, which needs energy. All potential unobserved probabilistic images considering as abstract entity we model by axiomatic probabilities of a random field. An observing planet models a specific triad of the field, while its probabilistic trajectory models Markov chain.

The observer sends discrete Yes-No $\downarrow \uparrow$ probing impulses acting on his Markov chain' imitating the image trajectory. These actions carry the impulses changing probabilities of the events in the specific triad. That also changes the impulse probability, reflecting the specific triad observation. These impulse probabilities virtually observe the running chain by multiple interactive acts. A probabilistic observation of at least two discrete impulses of the chain connects a minimum of three its random events in mutual correlation during the observing random time.

The Markov chain under such observation transforms into the Markov diffusion process [5].

The process's transitive probabilities define the Bayesian probabilities [4:77], whose sequential of priori-posteriori probabilities brings probabilistic impulse observation of the Markov diffusion process. Each observing No action $\downarrow$, in a sequence of these probabilities, virtually cuts entropy of the impulse correlation from the observing Markov diffusion process and transfers Yes action $\downarrow$ to the Bayes observing-process impulses. During the transition switching time, each impulse accumulates entropy spent on the interactive transition, sequentially increasing the entropy of the observing impulses. The entropy, cut from the Markov process decreases, while entropy enclosed within each impulse interactive action $\downarrow \uparrow$ increases.

Each following interactive impulse of the Bayes probabilities, observing the Markov process, maximizes the entropy of the Bayes observing-process impulses. The multiple Bayes observing impulses generate the secondary Markov diffusion, whose entropy increases, compared with that in the initial Markov process. Under such observations, the Markov diffusion evolves to a Lévy process [6]. The Lévy process's independent random increments model multiple observing impulses of the random bits. The Lévy-Khintchine decomposition [6:113-117] characterizes any Lévy process in terms of three components (the Lévy triplet) one of which is the Lévy measure. This measure describes the distribution of the Lévy process's potential jumps. Until any two time intervals of the process's independent random increments do not overlap, the jump absent and Lévy measure is zero. The overlap is a merge of the yes-no impulses, when the Lévy measure is non-zero-finite. The merging of the yes-no impulses indicates zero random bit.

When the Lévy measure becomes finite, the jumps have started. The jumps initiate a microprocess within the observing Lévy process, which releases the entropy uncertainty hidden in 
the cutting correlation, finally generating a non-random real bit. At the merging, the probability of the impulse carrying a bit is zero. It means there is no reality, defined by the interaction, when the microprocess starts. With zero probability of the start of the microprocess it is unpredictable, however it is developing an information process. The information process starts when the microprocess generates real qubit and/or bit. The considered path from emerging probabilistic impulse observation up to zero impulse probability identifies a virtual (uncertain) process covering hidden information, which precedes the starting information process. Even evolution is an interacting process.

\section{Essence of Information Process and the Intelligent Observer}

The Information process emerges within observing the random Lévy process, starting with unpredictable (probability zero) merge overlap of the yes-no impulses creating a random jump with conjugated entropy fractions beginning a microprocess.

The emerging microprocess runs a superposition and entanglement of the impulse yes-no conjugated entropy fractions. The fractions entangle during the time interval before space is formed, composed of two qubits and/or a bit, which set up a reversible logic within this impulse-created geometry.

When the impulse interacting actions curve the impulse space geometry, such interaction logically erases previous forms of the entangled space entropy, creating an asymmetrical logic bit as a logical Maxwell's demon.

With approaching probability of one, the impulse's attracting interaction captures energy of such a real/certain interactive action, which physically erases the entropy of bit logic.

The erasure costs the compensation of the high-quality energy of the physical (quantum) process with the entropy of lesser quality carrying the initial Markov diffusion.

This removes the causal entropy of the asymmetrical logic, bringing an asymmetrical information logical bit as a certain impulse bit. Such a bit is naturally extracted at a minimal-quality energy measure equivalent to entropy $\ln 2$. The virtual probabilistic (entropy) interaction becomes a real physical bit. The bit is memorized at the cost of Landauer's energy, working as a Maxwell's demon.

The memorized impulse includes information bits and free information, enclosing hidden information of the cutting correlation of the Markov-process impulses.

Statistical microthermodynamics describe the interacting energy of the microprocess ensemble. Multiple bits self-organize the information macroprocess, performing functions of Weller's bit-participator [2]. Along the multi-dimensional observation process, each piece of memorized information binds the reversible microprocess with the irreversible information macroprocess. The cutting entropy is automatically converting to information, conveying the process's logical information causality, certain logic, and complexity. The process's free information self-cooperates the bits in triple information units. The triplet units assemble an information network (IN), encoding the units in information geometrical structures enclosing the triplets' code. The IN triplets request the needed information, generating a logic of probing impulses, and sequentially cutting the observing-process entropy measure and encoding new information units in the hierarchical IN.

Information bits become a hierarchical measure of certain reality on the path from uncertainty.

The Observer's cognition assembles common units through multiple attractions in resonance loops at the forming IN-triplet hierarchy. The distributed cognitive logic self-controls encoding of the intelligence in a double helix coding structure (DSS).

The Observer's clock time intervals open access to external energy at each specific level of the IN multiple hierarchy, enabling memorization and encoding the hierarchy of these bits.

Multiple INs bind their ending triplets, enclosing Observer information, hierarchical reality, cognition, and intelligence.

The time, which starts the information process of the discrete information impulses, begins its Information Path Functional (IPF) integral information measure. 
The IPF integrates the multiple interactive dynamics in the finite bits, which observe and measure reality during the time integrating path to reality [7-9].

The minimax variation principle for the IPF [10] determines equations of information macrodynamics [11,12]. The IPF on the extreme trajectory of the information impulses is limited to measuring certain reality, while the impulse information measure density growing to infinity reaches an absolute and unobservable reality. The multiple interactive dynamics in finite bits, which observe and measure reality during the time integrating path to reality, integrate Interactive Integrated Information Dynamics (IIID). The IIID formalizes the information regularities of the observing random process and connects multi-level interactive reality with its information measures.

The intelligent Observer, self-reflective to the DSS logic, enables the reading and understanding of the message's meaning [13].

It is shown that the time and space of reality exists only as discrete units of information.

This brings the information measure to Wheeler's fundamental constant of nature [2].

The basic physical laws describe continuous reality.

The IIID describes the observing interactive dynamics of doublet-triplet logic the IN formation; integration of logic memory and cognition; the encoding of the intelligent Observer in triplet code, which further encodes physical information, cognition, intelligence including consciousness, and motor function. These enable the creation of an AI intellectual brain in the discrete reality.

\section{References}

1. Davies, P.; Gregersen, N.H. Information and the Nature of Reality: From Physics to Metaphysics; Cambridge Univ. Press: Cambridge, UK, 2011.

2. Wheeler, J.A. Hermann Weyl and Unity of Knowledge. Am. Sci. 1986, 74, 366-375.

3. Horgan, J. Why information can't be the basis of reality. Sci. Am. 2011,127, 1-7.

4. Kolmogorov, A.N. Foundations of the Theory of Probability; Courier Dover Publications: Chelsea, NY, USA, 1956.

5. Dynkin, E.B. Theory of Markov Processes; Prentice-Hall: Upper Saddle River, NY, USA, 1961.

6. Levy, P.P. Stochasic Processes and Brownian Movement; Deuxieme Edition: Paris, France, 1965.

7. Lerner, V.S. The boundary value problem and the Jensen inequality for an entropy functional of a Markov diffusion process. J. Math. Anal. Appl. 2009, 353, 154-160.

8. Lerner, V.S. The impulse cutoff an entropy functional measure on trajectories of Markov diffusion process integrating in information path functional. arXiv 2012, arXiv:1204.5513.

9. Lerner, V.S. Information Path from Randomness and Uncertainty to Information, Thermodynamics, and Intelligence of Observer. arXiv 2014, arXiv:1401.7041.

10. Lerner, V.S. Solution to the variation problem for information path functional of a controlled random process functional. J. Math. Anal. Appl. 2007, 334, 441-466.

11. Lerner, V.S. Information Systems Theory and Informational Macrodynamics: Review of the Main Results, IEEE Trans. Syst. Man Cybern. Part C Appl. Rev. 2007, 37, 1050-1066.

12. Lerner, V.S. Information Path Functional and Informational Macrodynamics; Nova Sc. Publ.: NY, USA, 2010.

13. Lerner, V.S. How Information Creates Its Observer. The Emergence of the Information Observer with Regularities; Nova Sc. Publ.: NY, USA, 2019.

(C) 2020 by the authors. Licensee MDPI, Basel, Switzerland. This article is an open access article distributed under the terms and conditions of the Creative Commons Attribution (CC BY) license (http://creativecommons.org/licenses/by/4.0/). 